\title{
Unintended facet fusions after Dynesys dynamic stabilization in patients with spondylolisthesis
}

\author{
Li-Yu Fay, MD, ${ }^{1-3}$ Wen-Cheng Huang, MD, PhD, ${ }^{1,2}$ Chih-Chang Chang, MD,, \\ Hsuan-Kan Chang, MD, ,,2 Tzu-Yun Tsai, MD, ,,5 Tsung-Hsi Tu, MD, , ${ }^{1,2}$ Ching-Lan Wu, MD, 2,6 \\ Henrich Cheng, MD, PhD, ${ }^{1-3}$ and Jau-Ching Wu, MD, PhD ${ }^{1-3}$
}

\begin{abstract}
1Department of Neurosurgery, Neurological Institute, Taipei Veterans General Hospital; ${ }^{2}$ School of Medicine, National Yang-Ming University; ${ }^{3}$ nstitute of Pharmacology, National Yang-Ming University; ${ }^{4}$ Department of Ophthalmology, National Taiwan University Hospital, College of Medicine, National Taiwan University; ${ }^{5}$ Department of Ophthalmology, Far Eastern Memorial Hospital, New Taipei City; ${ }^{6}$ Department of Radiology, Taipei Veterans General Hospital; and ${ }^{7}$ Taiwan International Graduate Program in Molecular Medicine, National Yang-Ming University and Academia Sinica, Taipei, Taiwan
\end{abstract}

\begin{abstract}
OBJECTIVE The pedicle screw-based Dynesys dynamic stabilization (DDS) has reportedly become a surgical option for lumbar spondylosis and spondylolisthesis. However, it is still unclear whether the dynamic construct remains mobile or eventually fuses. The aim of this study was to investigate the incidence of unintended facet arthrodesis after DDS and its association with spondylolisthesis.
\end{abstract}

METHODS This retrospective study was designed to review 105 consecutive patients with 1- or 2-level lumbar spondylosis who were treated with DDS surgery. The patients were then divided into 2 groups according to preexisting spondylolisthesis or not. All patients underwent laminectomies, foraminotomies, and DDS. The clinical outcomes were measured using visual analog scale (VAS) scores for back and leg pain, Japanese Orthopaedic Association (JOA) scores, and Oswestry Disability Index (ODI) scores. All medical records, including pre- and postoperative radiographs, CT scans, and MR images, were also reviewed and compared.

RESULTS A total of 96 patients who completed the postoperative follow-up for more than 30 months were analyzed. The mean age was $64.1 \pm 12.9$ years, and the mean follow-up duration was $46.3 \pm 12.0$ months. There were 45 patients in the spondylolisthesis group and 51 patients in the nonspondylolisthesis group. The overall prevalence rate of unintended facet fusion was $52.1 \%$ in the series of DDS. Patients with spondylolisthesis were older (67.8 vs 60.8 years, $p=0.007$ ) and had a higher incidence rate of facet arthrodesis $(75.6 \%$ vs $31.4 \%, p<0.001)$ than patients without spondylolisthesis. Patients who had spondylolisthesis or were older than 65 years were more likely to have facet arthrodesis (OR 6.76 and 4.82, respectively). There were no significant differences in clinical outcomes, including VAS back and leg pain, ODI, and JOA scores between the 2 groups. Furthermore, regardless of whether or not unintended facet arthrodesis occurred, all patients experienced significant improvement (all $p<0.05$ ) in the clinical evaluations.

CONCLUSIONS During the mean follow-up of almost 4 years, the prevalence of unintended facet arthrodesis was $52.1 \%$ in patients who underwent DDS. Although the clinical outcomes were not affected, elderly patients with spondylolisthesis might have a greater chance of facet fusion. This could be a cause of the limited range of motion at the index levels long after DDS.

https://thejns.org/doi/abs/10.3171/2018.8.SPINE171328

KEYWORDS lumbar spondylosis; Dynesys; dynamic stabilization; facet joints; arthrodesis; spondylolisthesis

$\mathrm{P}$ EDICLE screw-based dynamic stabilization systems have been accepted as an alternative to fusion devices in the surgical management of lumbar spondylosis. Common indications of these nonfusion instruments have included degenerative disc disease (DDD), severe spondylosis with spinal stenosis, recurrent disc hernia- tion, mild spondylolisthesis, and even short-segment deformity. ${ }^{3,8,11,12,20-25,28}$ The rationale of dynamic stabilization is to unload the disc, transfer the axial loading posteriorly, and provide controlled motion over the bridged segments. ${ }^{8}$ Although the outcomes are reportedly satisfactory, it is not uncommon to discover that the bridged segment demon-

ABBREVIATIONS DDD = degenerative disc disease; DDS = Dynesys dynamic stabilization; JOA = Japanese Orthopaedic Association; ODI = Oswestry Disability Index; $\mathrm{ROM}=$ range of motion; VAS = visual analog scale.

SUBMITTED March 2, 2018. ACCEPTED August 15, 2018.

INCLUDE WHEN CITING Published online December 7, 2018; DOI: 10.3171/2018.8.SPINE171328. 
strates only limited motion after a certain period of time postoperatively. Previous studies from our institution also had comparable clinical and radiological results, which demonstrated that patients who underwent Dynesys dynamic stabilization (DDS, Zimmer Spine) yielded satisfactory clinical improvement with modest segmental mobility during 2-5 years of follow-up..$^{7,8,12,28}$ However, it is not clear why these so-called dynamic, nonfusion devices eventually only allow limited range of motion (ROM) in vivo.

Previous studies from multiple independent institutions have demonstrated that a substantial portion of patients who had undergone DDS developed bone fusion over the facet joints. Theoretically, there was no violation of facets or bone grafts during the initial DDS surgery. However, elimination of the joint space of the bridged facets with solid bone growth in between was noticed on postoperative CT scans. ${ }^{6,16}$ Approximately half of the patients in one study developed facet arthrodesis. ${ }^{6}$ Even though unintended facet arthrodesis seemed to cause few adverse effects on the midterm clinical outcomes, the risk factors and long-term impact remain unknown. It was reasonable to assume that although dynamic stabilization was the goal, the construct ultimately became rigidly fixed. This issue would raise more concern when patients with spondylolisthesis, who had obvious instability, are treated with the dynamic stabilization.

The present study aimed to investigate the differences between patients with and without spondylolisthesis who were treated using the DDS system. The study retrospectively reviewed all the clinical and radiological outcomes of patients who underwent DDS, including postoperative CT scans for every patient. Thus, any postoperative degenerative changes over the facet joints would be evaluated in detail. Furthermore, this was the first report that specifically addressed the effectiveness and unintended facet arthrodesis after dynamic stabilization in patients with spondylolisthesis.

\section{Methods \\ Patient Population}

Consecutive patients with symptomatic lumbar spinal stenosis with or without spondylolisthesis who underwent posterior decompression and DDS in our service between 2010 and 2012 were included and retrospectively reviewed. Common symptoms of these patients included low-back pain, radicular pain, neurogenic claudication, and cauda equina syndrome. The offending pathologies included spinal stenosis caused by grade I spondylolisthesis, yellow ligament hypertrophy, and DDD. Patients were excluded if they had Meyerding grade II spondylolisthesis, coronal plane deformity, sagittal plane imbalance, severe facet degeneration, or disc collapse causing arthrodesis prior to the surgery. Most of the excluded patients were better suited for fusion surgery. Furthermore, patients with immobile segmental motion $\left(<3^{\circ}\right)$ on lateral dynamic radiographs would have been recommended for fusion procedures rather than the DDS. Patients who were lost to follow-up or did not complete evaluations at each time point were also excluded from analysis. This study was approved by the institutional ethics committee.

\section{Surgical Technique}

Patients were placed prone after induction of general anesthesia. Care was taken to ensure adequate lumbar lordosis, which was confirmed by lateral fluoroscopy prior to the surgery. The midline skin and fascia incisions were made for decompression with laminectomy and foraminotomy at the index level. Bilateral lateral recesses were widened using a Kerrison rongeur by removing the hypertrophic yellow ligaments and soft tissue. The nerve roots were then released from pressure and probed along through the neural foramen. Care was also taken to preserve the capsules of the facet joints during dissection. However, in some cases, the medial side of the hypertrophic facet joint was partially resected to achieve adequate decompression of the lateral recess. The joint spaces of the facets that were bridged were left intact without any violation or bone grafting.

Instrumentation with DDS was subsequently performed after decompression. Subcutaneous dissection was performed to widen the lateral aspects of the midline skin incision. Bilateral fascial incisions were then made for traversing Wiltse's plane to the junction of the transverse process and lateral side of the facet. The Dynesys pedicle screws were then placed under fluoroscopic guidance. Again, the facet joints were not exposed during screw placement. The sizes of the titanium alloy screws were estimated on preoperative CT scans and by pedicle sounding intraoperatively. To increase the purchase of these dynamic pedicle screws, it was recommended that removal or reinsertion of the pedicle screws should be avoided. After insertion of the pedicle screws, the elastic spacers made of polycarbonate urethane with customized lengths according to level were then mounted with the polyethylene-terephthalate tension cords. The elastic spacer was designed to absorb forces during extension, while the tension cord was designed to provide a pulling strength that could limit the extent of forward bending and thus could allow the patient's lumbar spine to move in a controlled ROM, including lateral bending and axial rotation. Theoretically, patients who undergo DDS should be stabilized in a neutrally lordotic position.

\section{Clinical Evaluation}

We used a clinical database at our institution that was built to prospectively collect the patients' follow-up data. At each time point, the planned clinical and radiological evaluations were performed during the clinic visits. Preoperative evaluation was done at the time the patient decided to undergo surgical intervention. Postoperative clinic visits at $1.5,3,6,12$, and 24 months after surgery and yearly thereafter were planned. Patients scored subjective questions themselves. Some patients required the assistance of our special study nurses. The objective assessments were done by the 2 special study nurses under supervision of the physicians.

\section{Radiological Evaluation}

Before surgery, all patients underwent anteroposterior and dynamic lateral radiography, CT scanning, and MRI for evaluation. Postoperative imaging evaluations included 
anteroposterior and dynamic lateral radiographs at $1.5,3$, 6,12 , and 24 months after surgery and every 12 months thereafter. Follow-up CT scanning and MRI were scheduled at approximately 18 and 24 months postoperatively. In the series, spondylolisthesis was classified by Meyerding grading. Patients with an unstable lumbar spine who considered undergoing DDS would not have spondylolisthesis worse than grade I. Patients with Meyerding grade II or higher spondylolisthesis were not considered candidates for DDS and were recommended to undergo fusion surgery.

After the DDS surgery, the patients underwent regular follow-up with lateral dynamic radiography for evaluation of ROM at the bridged levels. Unintended facet arthrodesis after DDS was defined as effacement of the facet joint space and continuous cortical calcification between the superior and inferior articular processes. Every DDS-bridged facet joint was evaluated on the CT scans, and the facet fusions of the index level(s) were confirmed by continuous bone growth causing effacement of the joint space demonstrated on CT scanning. This definition was the same as in previous publications on the issue of facet arthrodesis.

For evaluation of the segmental ROM at the index level(s), changes in the lordotic angles during flexion and extension were measured on lateral dynamic radiographs. Segmental ROM was defined as the difference in the Cobb angles of treated levels between flexion and extension. An immobile spine was defined as a segmental ROM of $\leq$ $3^{\circ}$. Theoretically, unintended facet arthrodesis after DDS would correlate with immobility (i.e., loss of segmental ROM) at the index level(s). All imaging studies were reviewed through the SmartIris digital medical imaging system (Taiwan Electronic Data Processing Co.). Two independent radiologists and 2 neurosurgeons reviewed the images, and the coauthors made the final decision if there was any ambiguity among interpretations.

\section{Statistical Analysis}

All statistical analyses were performed using SPSS (version 18 SPSS, Inc.). Independent t-tests and paired ttests were used for continuous variables, and the Fisher exact test was used for categorical data. Continuous variables are presented as the mean $\pm \mathrm{SD}$. The chi-square test was used for 2 variables relation verification; $p<0.05$ was considered to be statistically significant.

\section{Results}

Among the 105 consecutive patients who underwent DDS in this series, $96(91.4 \%)$ patients completed the 30-month clinical and radiological evaluations after surgery. The mean age was $64.1 \pm 12.9$ years at the time of surgery, and there were 45 females (46.9\%) and 51 males (53.1\%). The mean follow-up duration was $46.3 \pm 12.0$ months (Table 1).

Of the 96 patients analyzed, 37 (38.5\%) underwent 1-level and $59(61.5 \%)$ underwent 2-level DDS after decompression. There were $45(46.9 \%)$ patients with spondylolisthesis and 51 (53.1\%) patients without spondylolisthesis. A total of 155 disc levels and 310 facet joints were bridged, with 502 pedicle screws placed in the current
TABLE 1. Clinical and demographic characteristics

\begin{tabular}{cc}
\hline Characteristics & Value \\
\hline No. of patients & 96 \\
\hline Sex & \\
\hline Male & $51(53.1)$ \\
\hline Female & $45(46.9)$ \\
\hline Mean age, yrs & $64.1 \pm 12.9$ \\
\hline Mean follow-up, mos & $46.3 \pm 12.0$ \\
\hline Spondylolisthesis & $45(46.9)$ \\
\hline No. of bridged levels & $37(38.5)$ \\
\hline 1 & $59(61.5)$ \\
\hline 2 &
\end{tabular}

Values are presented as the number of patients (\%) unless stated otherwise. Mean values are presented \pm SD.

analysis. The distribution of index levels that underwent DDS varied from L2 to S1 (Fig. 1).

\section{Facet Arthrodesis and Segmental Mobility}

According to the follow-up CT scans, 50 (52.1\%) patients had unintended facet arthrodesis, and most of these fused facets allowed only limited mobility at the index level(s). The mean segmental ROM of these 50 patients with fused facets was $3.5^{\circ} \pm 3.3^{\circ}$, which was significantly smaller than that of patients without facet arthrodesis (mean ROM 5.7 $\pm 3.4^{\circ}, \mathrm{p}=0.001$ ) (Table 2).

The possible risk factors of the unintended facet arthrodesis were age and preexisting spondylolisthesis. For example, patients with facet arthrodesis were on average 10.8 years older than patients without facet arthrodesis $(69.3 \pm 8.2$ vs $58.5 \pm 14.7$ years, $\mathrm{p}<0.001)$. Among the 50 patients who developed postoperative facet arthrodesis, 34 (68.0\%) had spondylolisthesis. Thus, patients who were older than 65 years and those who had spondylolisthesis were more likely to develop facet arthrodesis after DDS surgery (OR 4.82 and 6.76, respectively; both $\mathrm{p}<0.001$ [Table 2]).

Unintended facet arthrodesis could negatively influence segmental mobility after DDS. A majority (72.0\%) of the patients who had facet arthrodesis (36 of 50 patients) were nearly immobile (segmental ROM $<3^{\circ}$ ) at the level(s) that underwent DDS. On the other hand, the 46 patients whose facets had no signs of fusion (no arthrodesis demonstrated on their CT scans) had a mean segmental ROM of $5.7^{\circ} \pm$ $3.4^{\circ}$. Also, less than one-quarter of them (23.9\%) had segmental ROM less than $3^{\circ}$ (Table 2). Patients who had facet arthrodesis tended to have higher rates of immobility after DDS in the stabilized lumbar segments than those who did not develop facet arthrodesis $(72.0 \%$ vs $23.9 \%$, p < 0.001$)$. However, patient sex and the number of bridged levels were similar between patients with and without facet arthrodesis. Examples of patients with and without postoperative facet arthrodesis are demonstrated in Figs. 2 and 3.

\section{Clinical Outcomes}

Results of the clinical evaluations, including back and leg pain visual analog scale (VAS), Oswestry Disability 
TABLE 2. Comparison of data between facet arthrodesis or not

\begin{tabular}{|c|c|c|c|c|}
\hline \multirow[b]{2}{*}{ Characteristics } & \multirow{2}{*}{$\begin{array}{l}\text { Total No. } \\
\text { of Patients }\end{array}$} & \multicolumn{2}{|c|}{ Facet Arthrodesis } & \multirow{2}{*}{$\begin{array}{c}p \\
\text { Value }\end{array}$} \\
\hline & & Yes & No & \\
\hline No. of patients & 96 & $50(52.1)$ & $46(47.9)$ & \\
\hline Mean age, yrs & & $69.3 \pm 8.2$ & $58.5 \pm 14.7$ & $<0.001^{*}$ \\
\hline $\mathrm{ROM}^{\circ}$ & & $3.5 \pm 3.3$ & $5.7 \pm 3.4$ & $0.001^{*}$ \\
\hline Spondylolisthesis & & & & $<0.001^{*}$ \\
\hline Yes & 45 & $34(68.0)$ & $11(23.9)$ & \\
\hline No & 51 & $16(32.0)$ & $35(76.1)$ & \\
\hline \multicolumn{5}{|l|}{ OR 6.76} \\
\hline Age $>65$ yrs & & & & $<0.001^{*}$ \\
\hline Yes & 50 & $35(70.0)$ & $15(32.6)$ & \\
\hline No & 46 & $15(30.0)$ & $31(67.4)$ & \\
\hline \multicolumn{5}{|l|}{ OR 4.82} \\
\hline Immobile & & & & $<0.001^{*}$ \\
\hline Yes & 47 & $36(72.0)$ & $11(23.9)$ & \\
\hline No & 49 & $14(28.0)$ & 35 (76.1) & \\
\hline \multicolumn{5}{|l|}{ Mean preop scores } \\
\hline VAS back pain & & $4.8 \pm 3.1$ & $5.7 \pm 2.6$ & 0.149 \\
\hline VAS leg pain & & $5.6 \pm 3.0$ & $6.2 \pm 2.8$ & 0.283 \\
\hline ODI & & $43.9 \pm 17.9$ & $47.0 \pm 22.0$ & 0.444 \\
\hline JOA & & $4.4 \pm 3.2$ & $4.3 \pm 3.3$ & 0.871 \\
\hline \multicolumn{5}{|l|}{$\begin{array}{l}\text { Mean 24-mo postop } \\
\text { scores }\end{array}$} \\
\hline VAS back pain & & $2.4 \pm 2.4$ & $2.0 \pm 2.3$ & 0.489 \\
\hline VAS leg pain & & $2.8 \pm 2.6$ & $2.0 \pm 2.4$ & 0.132 \\
\hline ODI & & $17.5 \pm 14.5$ & $17.4 \pm 13.3$ & 0.957 \\
\hline JOA & & $9.5 \pm 3.5$ & $10.1 \pm 3.0$ & 0.412 \\
\hline
\end{tabular}

Values are presented as the number of patients (\%) unless stated otherwise. Mean values are presented \pm SD.

* Statistically significant.

Index (ODI), and Japanese Orthopaedic Association (JOA) scores, at $3,6,12$, and 24 months postoperatively were all significantly improved compared with preoperative evaluations. Unintended facet arthrodesis did not have adverse impacts on the clinical outcomes. There were no significant differences between the group of patients with and those without facet arthrodesis in terms of back and leg pain VAS, ODI, and JOA scores preoperatively $(\mathrm{p}=0.149$, $0.283,0.444$, and 0.871 , respectively), or postoperatively ( $\mathrm{p}$ $=0.489,0.132,0.957$, and 0.412 , respectively). In general, all patients had significant improvement in back and leg pain VAS, ODI, and JOA scores regardless of unintended facet arthrodesis or not (Table 2 and Fig. 4).

\section{Comparison Between the Spondylolisthesis and Nonspondylolisthesis Groups}

The series of patients were further divided into the spondylolisthesis group and the nonspondylolisthesis group for comparison. Patients in the spondylolisthesis group were on average 7 years older than those without spondylolisthesis $(\mathrm{p}=0.007)$. The prevalence rates of unintended facet arthrodesis were higher in the patients with spondylolisthesis $(75.6 \%$ vs $31.4 \%, \mathrm{p}<0.001)$ than those without. The distribution of sex and number of bridged levels was similar in the 2 groups (Table 3 ).

Furthermore, patients with spondylolisthesis had a higher chance of immobility at the indexed segments after DDS (62.2\% vs 37.3\% for patients without spondylolisthesis, $\mathrm{p}=0.012$ ). However, both groups had similarly significant improvements in the clinical outcomes, including VAS back and leg pain, and disability and functional scores after surgery (Fig. 4 and Table 3 ).

\section{Complications}

There was $1(1.0 \%)$ iatrogenic durotomy during the operation. The dura was closed primarily with sutures and augmented with DURAFORM (DePuy Synthes). This patient did not have any new neurological symptoms after surgery or during follow-up. There were no deep wound infections in the series. No other secondary surgery, including revision for malpositioned or loosened screws, revision to fusion constructs, or decompression for stenosis caused by adjacent-segment disease, was performed during the follow-up.

\section{Discussion}

The current study analyzed 96 patients with lumbar spondylosis and stenosis who underwent decompression and stabilization with DDS. A total of 155 disc levels and 310 facet joints were bridged with DDS, with the aim of preserving segmental mobility instead of fusion. However, during the average follow-up of approximately 4 years, we surprisingly found facet arthrodesis in over half (52.1\%) of our patients. Unintended facet fusion was identified on CT scans during follow-up, and $49.0 \%$ of patients (47 of 96) had little segmental mobility that allowed less than $3^{\circ}$ segmental ROM at the index level(s). In other words, only $51.0 \%$ of the patients who underwent DDS eventually had preserved segmental mobility in their lumbar spine after 4 years. Nevertheless, the overall clinical outcomes of patients in the current series remained satisfactory regardless of facet fusion or not. The study also indicated that spondylolisthesis and age older than 65 years could be risk factors. Although the long-term consequences of facet arthrodesis remained elusive, it hardly affected the clinical improvements in this series up to 4 years of follow-up. This observation implied that the fused facets after DDS might have contributed to maintenance of the clinical improvement, especially in patients with mild instability. The initial stabilization effect of DDS could have provided just enough strength that was necessary to eliminate the instability in patients with spondylolisthesis.

This study was the first to address age and spondylolisthesis in association with the unintended facet arthrodesis after DDS. The finding, unintended facet arthrodesis, provided a reasonable explanation for the limited segmental ROM long after DDS, which our group previously reported. ${ }^{6}$ Such types of dynamic stabilization devices appeared to have acted more like a fixation device in longer-term follow-up. The claimed design rationale of motion preservation might eventually be fortuitous. However, the decelerated fixation effect that finally leads to fusion might be useful and adequate in many of the patients with lumbar spondylosis. Unintended facet arthrodesis had not 
Fay et al.
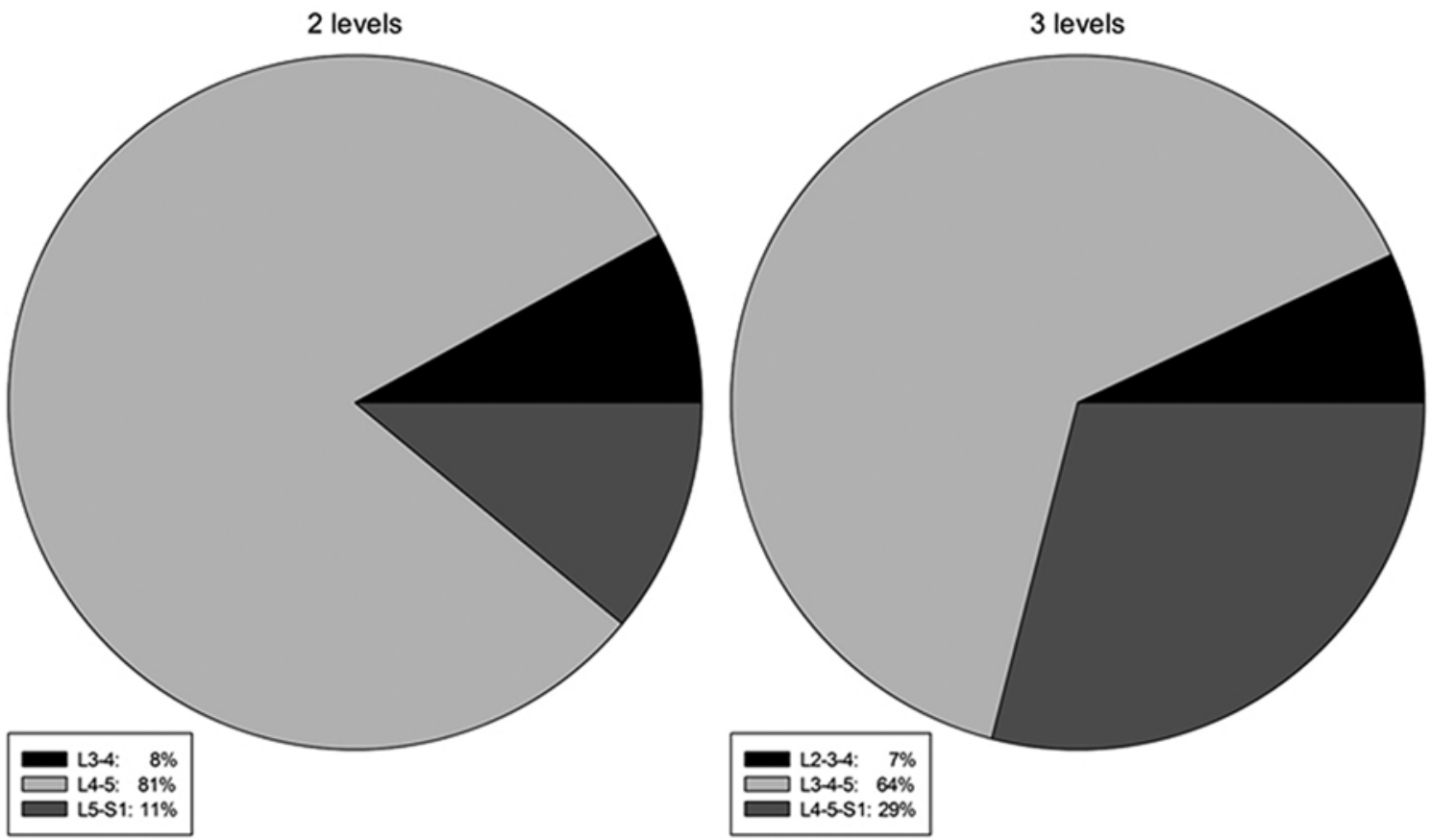

FIG. 1. Pie charts demonstrating the distribution of the index levels that were treated using DDS.

been reported until our group's report published in $2016 .{ }^{6}$ In the previous study, we collected 70 patients with 1- or 2-level lumbar spondylosis who underwent laminectomy and DDS. Among them, 38 (54.3\%) patients were found to have facet arthrodesis at the 2-year follow-up, and patients who had unintended facet arthrodesis were older than those who did not (68.3 vs 58.5 years, $\mathrm{p}=0.009$ ). Furthermore, there was a positive correlation between facet arthrodesis and segmental immobility. Similarly, the clinical outcomes were not affected.
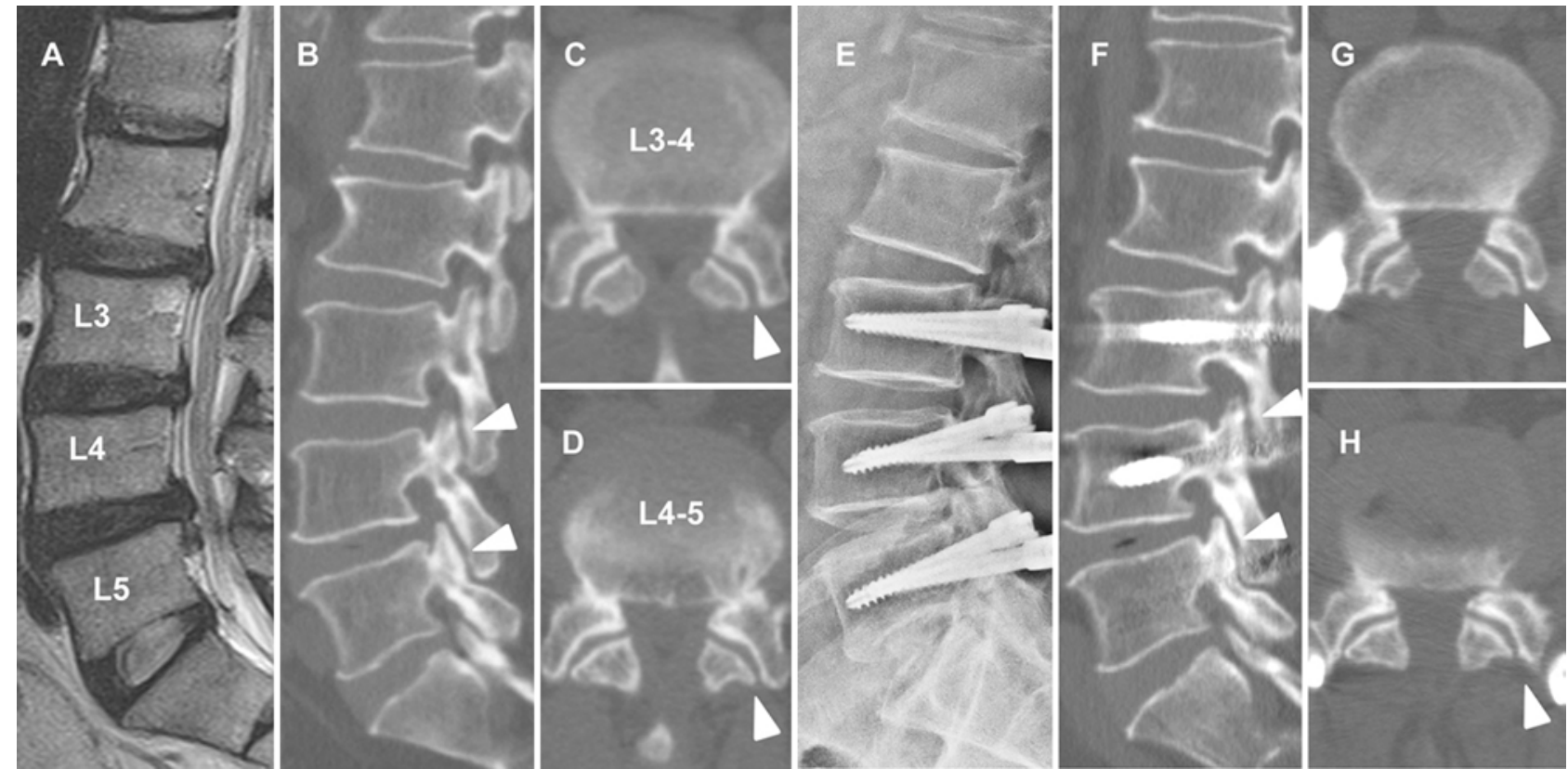

FIG. 2. Serial images obtained in a 68-year-old man who required lumbar spine surgery. A: Preoperative sagittal MR image. There was spinal stenosis at L3-5. B: Preoperative CT sagittal view of the left facet joints (arrowheads). C and D: Preoperative axial CT scan of $L 3-4$ and $L 4-5$. The joint spaces are visible (arrowheads). E: Postoperative lateral radiograph. F: Postoperative sagittal CT scan of the left facet joints (arrowheads). G and H: Postoperative axial CT of L3-4 and L4-5. The joint spaces are visible (arrowheads). 

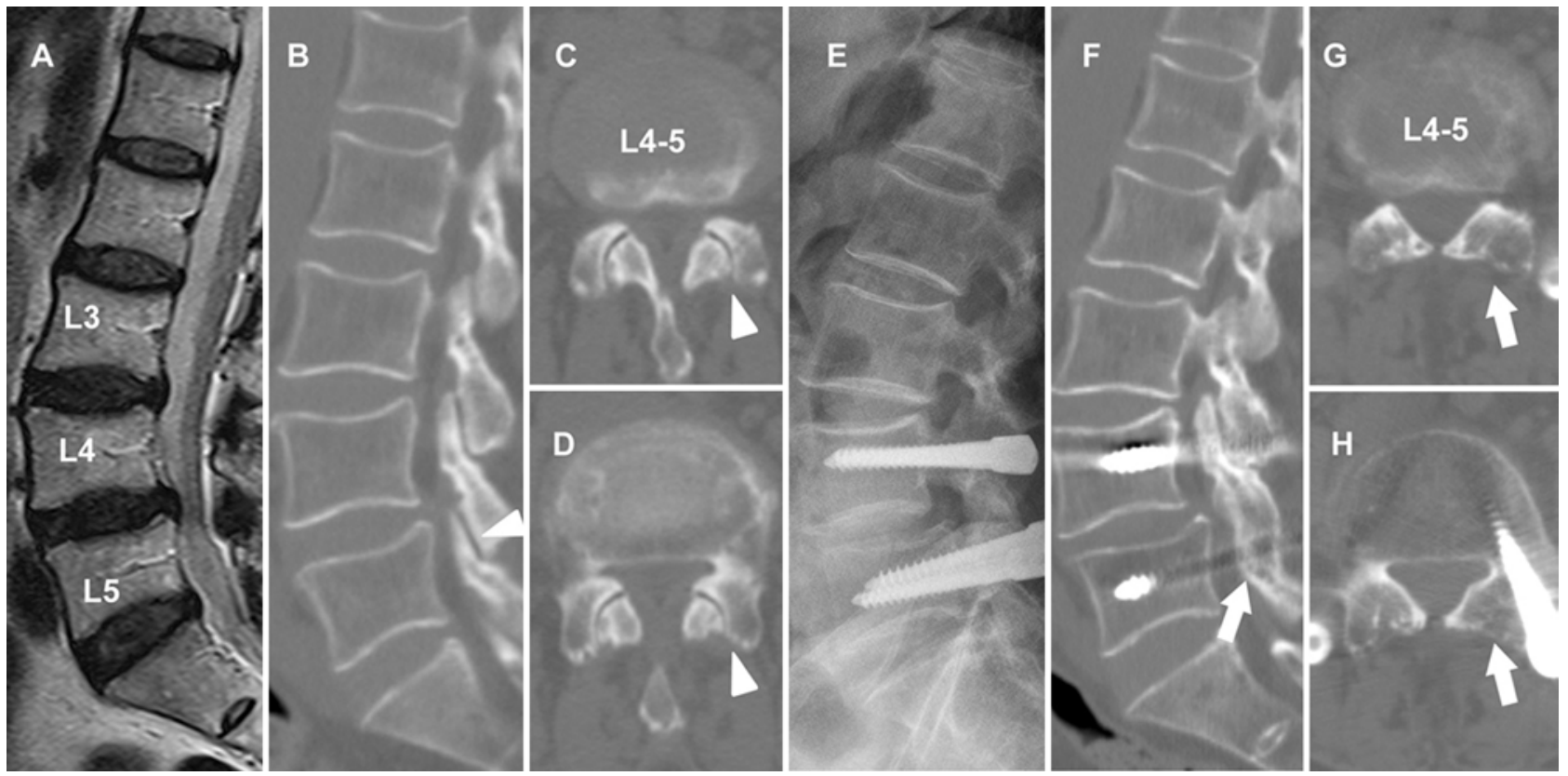

FIG. 3. Serial images obtained in a 59-year-old woman who needed lumbar spine surgery. A: Preoperative sagittal MR image. Spinal stenosis and spondylolisthesis are seen at L4-5. B: Preoperative sagittal CT scan of the left facet joints (arrowhead). C and D: Preoperative axial CT scans of different slices of L4-5. The joint spaces (arrowheads) were not changed. E: Postoperative lateral plain radiograph. F-H: Postoperative sagittal CT scan of the left facet joints (F) and postoperative axial CT scans of different cuts of $L 4-5$ ( $G$ and $H)$. The joint spaces were fused (arrow).

It remained elusive why the unintended facet fusion was more common in patients with spondylolisthesis after DDS. Although the current study design could only describe rather than explain the phenomenon of facet arthrodesis, there are several possible causes, including age, instability, and the natural disease course. Patients with spondylolisthesis were on average 7 years older than those without, and they might have more ongoing degeneration that could eventually lead to fusion than those who had no spondylolisthesis. The unintended fusion of facets has been reported before, and the study also demonstrated that older patients (age $>60$ years) had a 12 times higher risk of facet fusion than younger patients. The higher chances of facet arthrodesis found in the elderly in the studies could indicate that degeneration and spondylosis at a later stage might contribute to the facet fusion. ${ }^{6}$ Furthermore, spondylolisthesis per se could refer to more instability and inflammation. Therefore, the unintended arthrodesis could also imply the need for stabilization with instrumentation. Lee et al. also demonstrated facet joint degeneration at the instrumented and cranial adjacent levels..$^{16}$ Of the 37 patients reported with L4-5 spondylosis, 15 received DDS, and 22 received fusion surgery. Facet joint degeneration was worse in the fusion group, but DDS did not prevent degeneration. Although the back and leg pain scores were not significantly different between the 2 groups, the improvements in ODI functional scores were significantly better in patients who received DDS. The authors also found that the composition of sex and spondylotic levels were not different in the patients with or without postoperative facet arthrodesis. Patients with unintended facet arthrodesis were a mean 10 years older than those without it. These results are consistent with our studies. ${ }^{6}$ Older patients likely have degenerative conditions of the spine, and thus the segments are more prone to fuse (Table 2).

Ideally, DDS was meant to preserve segmental spinal motion, at least partially. Many reports have demonstrated overall preserved segmental mobility. $3,5,9,11,19,21,25,26$ Schnake et al. reported that more than half $(54.2 \%)$ of the 24 patients who underwent DDS demonstrated relatively limited segmental motion $\left(<3^{\circ}\right)$ at the 2-year follow-up..$^{24}$ Schaeren et al. also conducted a study with 4 years of follow-up in 2008, in which the 26 patients who underwent DDS demonstrated similar results. ${ }^{23}$ Our previous study also demonstrated that 38 patients had limited mean motion $\left(<3^{\circ}\right)$ at 3.5 years after DDS surgery. ${ }^{8}$ Our finding of unintended facet arthrodesis could be accounted for by the limited motion after DDS. ${ }^{6}$ The study collected 70 patients who underwent DDS surgery, and 38 (54.3\%) had facet arthrodesis at the 29.9-month follow-up. Furthermore, almost three-fourths of the 38 patients $(71.1 \%)$ demonstrated an immobile lumbar spine. The facet arthrodesis probably would contribute to subsequent limited motion at longterm follow-up.

The surgical indications for placement of the DDS in the present study were symptomatic lumbar spinal stenosis with the coexistence of at least 1 of the following pathologies: grade I spondylolisthesis, yellow ligament hypertrophy, multiple recurrent disc herniations, or DDD. For these patients, DDS aimed to shift the disc load posteriorly and augment segmental stability at the index level. However, we have to admit that the choice between pedicle screwbased dynamic stabilization and pedicle screw fixation and fusion was indeed sometimes a gray area. In our series, 

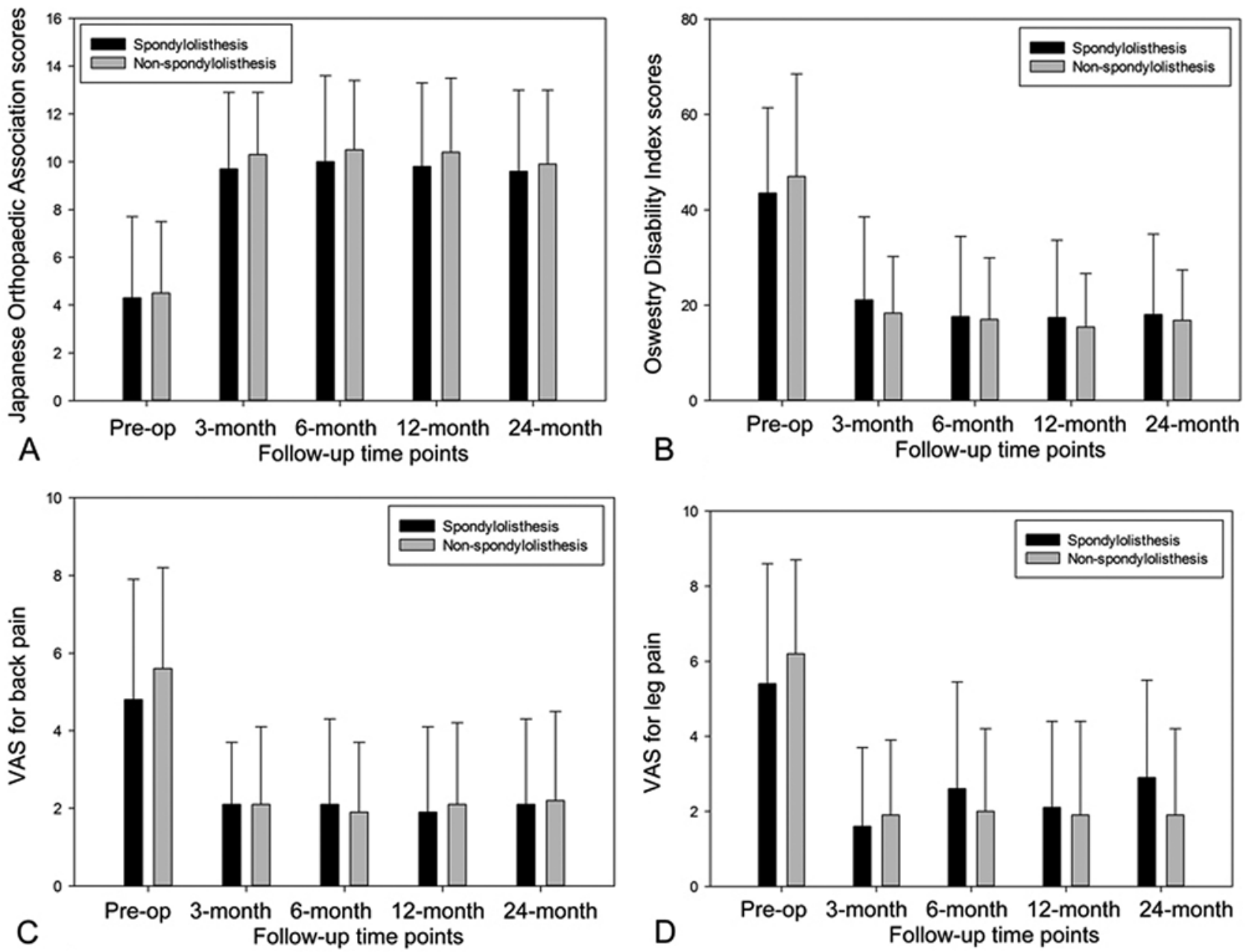

FIG. 4. Bar graphs showing the JOA scores (A), ODI scores (B), and the VAS scores for back pain (C) and leg pain (D) at each time point of observation. There were significant improvements postoperatively when compared with that assessed preoperatively $(p<0.05$ at each time point). The numbers indicate the mean value of each score.

patients with Meyerding grade II spondylolisthesis, coronal plane deformity, sagittal plane imbalance, severe facet degeneration, or disc collapse causing arthrodesis prior to the surgery were excluded from receiving DDS. Most of these excluded patients were better suited for fusion surgery. Furthermore, patients with immobile segmental motion $\left(<3^{\circ}\right)$ on lateral dynamic radiographs would have been recommended for fusion procedures rather than DDS. Surprisingly, in our series of DDS, ${ }^{4,6,12-14,28,29}$ a substantial portion of the patients who underwent dynamic stabilization ended up with little segmental mobility and were found to have the facets fused. Therefore, the present study was designed to demonstrate that spondylolisthesis could be a risk factor of the limited segmental mobility found after DDS, although DDS was originally designed for preservation of motion and avoidance of fusion.

There were few differences in clinical improvement among patients with or without facet arthrodesis. Furthermore, both groups of patients had satisfactory clinical outcomes. Facet arthrodesis could be regarded as a kind of lumbar posterolateral fusion that was targeted at facets. The DDS patients who developed no facet arthrodesis were stabilized only with the titanium alloy pedicle screws connected with elastic spacers. Patients who had normal facet joints were very likely maintaining their spinal mobility afterward. Although the impact of facet arthrodesis is not yet clear, the clinical results could be strong support for the efficacy and durability of DDS.

To further analyze the correlation between spondylolisthesis and facet arthrodesis, we divided the patients by age. There was a positive correlation between spondylolisthesis and facet arthrodesis in both age groups (Tables 2 and 3 ). Moreover, the odds ratio between old age or having an unstable spine and facet arthrodesis were calculated. The positive correlation and odds ratio were confirmed (4.82 vs 6.76).

Spinal fusion has frequently been considered as a very effective surgical option for lumbar spondylosis, particularly in patients with instability. However, there are multiple concerns about adjacent-segment degeneration (ASD) after lumbar fusion. ${ }^{1,2,10,17,18,27}$ Since DDS is another option to stabilize the spine and preserve the limited motion in the degenerative spine, theoretically it could lower the chances of ASD. We conducted studies to compare lumbar fusion 
TABLE 3. Comparison of data between spondylolisthesis and nonspondylolisthesis group

\begin{tabular}{|c|c|c|c|c|}
\hline \multirow[b]{2}{*}{ Characteristics } & \multirow[b]{2}{*}{ Overall } & \multicolumn{2}{|c|}{ Spondylolisthesis } & \multirow{2}{*}{$\begin{array}{c}\mathrm{p} \\
\text { value }\end{array}$} \\
\hline & & Yes & No & \\
\hline No. of patients & 96 & $45(46.9)$ & $51(53.1)$ & \\
\hline Mean age, yrs & $64.1 \pm 12.9$ & $67.8 \pm 9.4$ & $60.8 \pm 14.7$ & $0.007^{*}$ \\
\hline Facet arthrodesis & & & & $<0.001^{*}$ \\
\hline Yes & 50 & $34(75.6)$ & $16(31.4)$ & \\
\hline No & 46 & $11(24.4)$ & $35(68.6)$ & \\
\hline Sex & & & & 0.306 \\
\hline Male & 51 & $21(46.7)$ & $30(58.8)$ & \\
\hline Female & 45 & $24(53.3)$ & $21(41.2)$ & \\
\hline Bridged level(s) & & & & 0.362 \\
\hline 1 & 37 & $16(35.6)$ & $21(41.2)$ & \\
\hline 2 & 59 & $29(64.4)$ & $30(58.8)$ & \\
\hline Immobile (postop) & & & & $0.012^{*}$ \\
\hline Yes & 47 & $28(62.2)$ & $19(37.3)$ & \\
\hline No & 49 & $17(37.8)$ & $32(62.7)$ & \\
\hline Segmental ROM, ${ }^{\circ}$ & & & & 0.531 \\
\hline Mean & $4.6 \pm 3.5$ & $4.3 \pm 4.1$ & $4.8 \pm 2.9$ & \\
\hline Range & $0-17$ & $0-17$ & $0-13$ & \\
\hline \multicolumn{5}{|l|}{ Mean preop scores } \\
\hline VAS back pain & $5.3 \pm 2.9$ & $4.9 \pm 3.2$ & $5.6 \pm 2.7$ & 0.229 \\
\hline VAS leg pain & $5.9 \pm 2.3$ & $5.4 \pm 3.3$ & $6.3 \pm 2.6$ & 0.178 \\
\hline ODI & $45.4 \pm 19.9$ & $43.6 \pm 18.0$ & $47.0 \pm 21.6$ & 0.395 \\
\hline JOA & $4.4 \pm 3.2$ & $4.3 \pm 3.5$ & $4.5 \pm 3.0$ & 0.774 \\
\hline \multicolumn{5}{|l|}{$\begin{array}{l}\text { Mean } 24 \text {-mo postop } \\
\text { scores }\end{array}$} \\
\hline VAS back pain & $2.2 \pm 2.3$ & $2.2 \pm 2.3$ & $2.2 \pm 2.4$ & 0.944 \\
\hline VAS leg pain & $2.4 \pm 2.5$ & $3.0 \pm 2.6$ & $1.9 \pm 2.4$ & 0.053 \\
\hline ODI & $17.5 \pm 13.8$ & $18.2 \pm 17.0$ & $16.8 \pm 10.7$ & 0.666 \\
\hline JOA & $9.8 \pm 3.3$ & $9.6 \pm 3.4$ & $9.9 \pm 3.1$ & 0.721 \\
\hline
\end{tabular}

with dynamic stabilization with an average follow-up of more than 2 years. ${ }^{14,15}$ Kuo et al. first examined 86 patients with L4-5 spondylolisthesis and demonstrated that the clinical and radiological outcomes of DDS were similar to those of minimally invasive transforaminal lumbar interbody fusion (mi-TLIF) for grade I spondylolisthesis after approximately 2.5 years postoperatively. Although DDS might be an alternative to standard arthrodesis in mild lumbar spondylolisthesis, the dynamic pedicle screws could have other issues like wearing and loosening in long-term follow-up. Another study aimed to address the discrepancy on the incidence of ASD between the DDS and mi-TLIF groups (dynamic stabilization vs fusion). Although the clinical outcomes were similar in both groups, by radiographic evaluations, there was a lower chance of disc degeneration in the adjacent levels of the patients who had undergone DDS. However, other radiological signs of ASD, including instability, endplate degeneration, and
ROM, were similar between the 2 groups. Although none of the patients in the series required secondary surgery, a longer follow-up and a larger number of patients would be necessary to corroborate the protective effect of DDS against ASD. ${ }^{15}$ The current study aimed specifically to investigate the differences among patients with and without spondylolisthesis after dynamic stabilization and thus did not include patients who underwent lumbar fusions. It remains elusive how spondylolisthesis might affect the theoretical benefit of DDS in the amelioration of ASD. The results indicated that approximately $75 \%$ of the patients who had DDS surgery for spondylolisthesis eventually ended up with facet arthrodesis. However, future research is needed to investigate the differences of ASD among these dynamically stabilized, or fused, patients with those who had mi-TLIF for arthrodesis in the beginning.

There are limitations to the current study. This was a retrospective consecutive study of 105 patients who underwent DDS surgery. Many of those who had spondylolisthesis also had extraordinarily severe facet degeneration and hypertrophic ligaments. It remains elusive whether the facet arthrodesis led to less ROM or vice versa. The merit of the study was that all facets were evaluated using CT scans for confirmation of the facet fusion. Obviously, longer-term follow-up and multivariate analysis are necessary to investigate the causes of the facet fusion. Also, a larger number of patients is necessary to clarify the long-term clinical effects. Nevertheless, DDS seemed to be a reasonable option for surgical intervention.

\section{Conclusions}

During the mean follow-up of almost 4 years, unintended facet arthrodesis had an overall prevalence rate of $52.1 \%$ in patients who underwent DDS. Although the clinical outcomes were not affected, elderly patients with spondylolisthesis might have a higher chance of facet fusion. This could be a cause of the limited range of motion at the index levels long after DDS.

\section{References}

1. Bassewitz H, Herkowitz H: Lumbar stenosis with spondylolisthesis: current concepts of surgical treatment. Clin Orthop Relat Res (384):54-60, 2001

2. Bridwell KH, Sedgewick TA, O’Brien MF, Lenke LG, Baldus $\mathrm{C}$ : The role of fusion and instrumentation in the treatment of degenerative spondylolisthesis with spinal stenosis. J Spinal Disord 6:461-472, 1993

3. Cakir B, Ulmar B, Koepp H, Huch K, Puhl W, Richter M: [Posterior dynamic stabilization as an alternative for dorsoventral fusion in spinal stenosis with degenerative instability.] Z Orthop Ihre Grenzgeb 141:418-424, 2003 (Ger)

4. Cheng YW, Chang PY, Wu JC, Chang CC, Fay LY, Tu TH, et al: Pedicle screw-based dynamic stabilization and adjacentsegment disease. J Neurosurg Spine 26:405-406, 2017 (Letter)

5. Di Silvestre M, Lolli F, Bakaloudis G, Parisini P: Dynamic stabilization for degenerative lumbar scoliosis in elderly patients. Spine (Phila Pa 1976) 35:227-234, 2010

6. Fay LY, Chang PY, Wu JC, Huang WC, Wang CH, Tsai TY, et al: Dynesys dynamic stabilization-related facet arthrodesis. Neurosurg Focus 40(1):E4, 2016

7. Fay LY, Wu JC, Tsai TY, Tu TH, Wu CL, Huang WC, et al: 
Intervertebral disc rehydration after lumbar dynamic stabilization: magnetic resonance image evaluation with a mean followup of four years. Adv Orthop 2013:437570, 2013

8. Fay LY, Wu JC, Tsai TY, Wu CL, Huang WC, Cheng H: Dynamic stabilization for degenerative spondylolisthesis: evaluation of radiographic and clinical outcomes. Clin Neurol Neurosurg 115:535-541, 2013

9. Freudiger S, Dubois G, Lorrain M: Dynamic neutralisation of the lumbar spine confirmed on a new lumbar spine simulator in vitro. Arch Orthop Trauma Surg 119:127-132, 1999

10. Glassman SD, Carreon LY, Djurasovic M, Dimar JR, Johnson JR, Puno RM, et al: Lumbar fusion outcomes stratified by specific diagnostic indication. Spine J 9:13-21, 2009

11. Grob D, Benini A, Junge A, Mannion AF: Clinical experience with the Dynesys semirigid fixation system for the lumbar spine: surgical and patient-oriented outcome in 50 cases after an average of 2 years. Spine (Phila Pa 1976) 30:324-331, 2005

12. Ko CC, Tsai HW, Huang WC, Wu JC, Chen YC, Shih YH, et al: Screw loosening in the Dynesys stabilization system: radiographic evidence and effect on outcomes. Neurosurg Focus 28(6):E10, 2010

13. Kuo CH, Chang PY, Tu TH, Fay LY, Chang HK, Wu JC, et al: The effect of lumbar lordosis on screw loosening in Dynesys dynamic stabilization: four-year follow-up with computed tomography. BioMed Res Int 2015:152435, 2015

14. Kuo CH, Chang PY, Wu JC, Chang HK, Fay LY, Tu TH, et al: Dynamic stabilization for L4-5 spondylolisthesis: comparison with minimally invasive transforaminal lumbar interbody fusion with more than 2 years of follow-up. Neurosurg Focus 40(1):E3, 2016

15. Kuo CH, Huang WC, Wu JC, Tu TH, Fay LY, Wu CL, et al: Radiological adjacent-segment degeneration in L4-5 spondylolisthesis: comparison between dynamic stabilization and minimally invasive transforaminal lumbar interbody fusion. J Neurosurg Spine 29:250-258, 2018

16. Lee SE, Jahng TA, Kim HJ: Facet joint changes after application of lumbar nonfusion dynamic stabilization. Neurosurg Focus 40(1):E6, 2016

17. Maeda T, Buchowski JM, Kim YJ, Mishiro T, Bridwell KH: Long adult spinal deformity fusion to the sacrum using rhBMP-2 versus autogenous iliac crest bone graft. Spine (Phila Pa 1976) 34:2205-2212, 2009

18. Mannion RJ, Nowitzke AM, Wood MJ: Promoting fusion in minimally invasive lumbar interbody stabilization with low-dose bone morphogenic protein-2-but what is the cost? Spine J 11:527-533, 2011

19. Nockels RP: Dynamic stabilization in the surgical management of painful lumbar spinal disorders. Spine (Phila Pa 1976) 30 (16 Suppl):S68-S72, 2005

20. Panjabi MM: The stabilizing system of the spine. Part I. Function, dysfunction, adaptation, and enhancement. J Spinal Disord 5:383-397, 1992

21. Putzier M, Schneider SV, Funk J, Perka C: [Application of a dynamic pedicle screw system (DYNESYS) for lumbar segmental degenerations-comparison of clinical and radiological results for different indications.] Z Orthop Ihre Grenzgeb 142:166-173, 2004 (Ger)
22. Putzier M, Schneider SV, Funk JF, Tohtz SW, Perka C: The surgical treatment of the lumbar disc prolapse: nucleotomy with additional transpedicular dynamic stabilization versus nucleotomy alone. Spine (Phila Pa 1976) 30:E109-E114, 2005

23. Schaeren S, Broger I, Jeanneret B: Minimum four-year follow-up of spinal stenosis with degenerative spondylolisthesis treated with decompression and dynamic stabilization. Spine (Phila Pa 1976) 33:E636-E642, 2008

24. Schnake KJ, Schaeren S, Jeanneret B: Dynamic stabilization in addition to decompression for lumbar spinal stenosis with degenerative spondylolisthesis. Spine (Phila Pa 1976) 31:442-449, 2006

25. Schwarzenbach O, Berlemann U, Stoll TM, Dubois G: Posterior dynamic stabilization systems: DYNESYS. Orthop Clin North Am 36:363-372, 2005

26. Stoll TM, Dubois G, Schwarzenbach O: The dynamic neutralization system for the spine: a multi-center study of a novel non-fusion system. Eur Spine J 11 (Suppl 2):S170-S178, 2002

27. Weinstein JN, Lurie JD, Tosteson TD, Zhao W, Blood EA, Tosteson AN, et al: Surgical compared with nonoperative treatment for lumbar degenerative spondylolisthesis. Fouryear results in the Spine Patient Outcomes Research Trial (SPORT) randomized and observational cohorts. J Bone Joint Surg Am 91:1295-1304, 2009

28. Wu JC, Huang WC, Tsai HW, Ko CC, Wu CL, Tu TH, et al: Pedicle screw loosening in dynamic stabilization: incidence, risk, and outcome in 126 patients. Neurosurg Focus 31(4):E9, 2011

29. Yeh MY, Kuo CH, Wu JC, Huang WC, Tu TH, Fay LY, et al: Changes of facet joints after dynamic stabilization: continuous degeneration or slow fusion? World Neurosurg 113:e45-e50, 2018

\section{Disclosures}

The authors report no conflict of interest concerning the materials or methods used in this study or the findings specified in this paper.

\section{Author Contributions}

Conception and design: JC Wu, Fay, Huang, Cheng. Acquisition of data: CC Chang, HK Chang, Tu, CL Wu. Analysis and interpretation of data: Fay, Huang, CC Chang, HK Chang, Tu. Drafting the article: JC Wu, Fay. Critically revising the article: JC Wu. Reviewed submitted version of manuscript: Huang. Statistical analysis: Tsai. Study supervision: Cheng.

\section{Correspondence}

Jau-Ching Wu: Neurological Institute, Taipei Veterans General Hospital, Taipei, Taiwan. jauching@gmail.com. 